\title{
Archéopages
}

Archéopages

Archéologie et société

Hors-série 1 | 2008

Construction $^{s}$ de l'archéologie

\section{Archéologie et diversité culturelle}

\author{
Luc Jallot
}

\section{(2) OpenEdition}

\section{Journals}

Édition électronique

URL : https://journals.openedition.org/archeopages/863

DOI : 10.4000/archeopages.863

ISSN : 2269-9872

\section{Éditeur}

INRAP - Institut national de recherches archéologiques préventives

Édition imprimée

Date de publication : 1 février 2008

Pagination : 84-91

ISSN : 1622-8545

\section{Référence électronique}

Luc Jallot, «Archéologie et diversité culturelle », Archéopages [En ligne], Hors-série 1 | 2008, mis en ligne le 01 février 2008, consulté le 02 mars 2023. URL : http://journals.openedition.org/archeopages/ 863 ; DOI : https://doi.org/10.4000/archeopages.863 
pertinents pour expliquer la genèse des communautés israélites des collines de Galilée, au cours des $\mathrm{II}^{\mathrm{e}}$ et $\mathrm{I}^{\mathrm{er}}$ millénaires avant notre ère. Dans un premier temps, il y a eu acte de violence étatique, les Arabes Shuwa étant les descendants des pasteurs nomades arabes expulsés de la vallée du Nil par les armées mameloukes, les Israélites étant les descendants des expulsés d'Égypte. Puis est apparue une phase migratoire plus ou moins longue, avec un mouvement vers le lac Tchad dans un cas, et des pérégrinations dans le désert du Sinaï dans l'autre. On observe ensuite une infiltration de petits groupes aux destins divers qui développèrent un sens de collectivité de destin face à l'adversité. Enfin, il y eut prise de pouvoir local. Les données archéologiques sous forme de carte d'implantation des sites, plans des structures d'habitat et des enclos à bétail ont alors été utilisées pour choisir les hypothèses les plus plausibles.

Conclusion. L'espace limité de cet article ne permet pas une analyse plus nuancée de l'évolution de l'ethnoarchéologie. Celle-ci est passée de la pratique des « discours édifiants» portant sur les limitations des interprétations archéologiques à des procédures de terrain ambitieuses, impliquant des programmes de longue durée. De fait, l'ethnoarchéologie - en mettant l'accent sur l'usage et la manipulation de la culture matérielle - a enrichi les pratiques archéologiques d'une puissante source d'inspiration.

Audouze F. (dir.) 1992 : Ethnoarchéologie: Justification, problèmes, Limites, Rencontres internationales d'archéologie et d'histoire d'Antibes, Juan-les-Pins, Éditions ApDCA.

ClARK J. D. 1968: «Studies of Hunter-gatherers as an aid to the interpretation of prehistoric societies", in R. B. Lee and I. DeVore (dir.), Man the Hunter, Chicago, Aldine, p. 276-28o.

David N., Kramer C. 2001: Ethnoarchaeology in Action, Cambridge, Cambridge University Press.

FinKelstein I. 1988: The Archaeology of Israelite Settlement, Jerusalem, Israel Exploartion Society.

Hodder I. 1982: Symbols in Action, Cambridge, Cambridge University Press.

Holl A. F. C. 2002: The Land of Houlouf: Genesis of a Chadic Chiefdom (BC190o-AD180o), Ann Arbor, The University of Michigan Museum of Anthropology.

Holl A. F. C. 2004 : Ethnoarchaeology of Shuwa-arab Settlements, Lanham, Lexington Books.

Levtzion N., Hopkins J. F. P. (dir.) 1981: Corpus of Early Arabic Sources for West African History, Cambridge, Cambridge University Press

LEVY E. L., Holl A. F. C. 2002 : «Migrations, Ethnogenesis, and Settlement Dynamics: Israelites in Iron Age Canaan and ShuwaArab in the Chad Basin ", Journal of Anthropological Archaeology, $\mathrm{n}^{\circ} 21, \mathrm{p} .83-118$

\section{Archéologie et diversité culturelle}

\author{
Luc Jallot \\ Inrap, UMR 5140, Archéologie des sociétés méditerranéennes, \\ milieu, territoire, civilisations
}

C'est en opposant les singularités qu'on comprend la richesse de l'universel. L'humanité n'est pas une fourmilière mais l'expression d'une diversité... Le fait d'être de quelque part donne conscience que chaque homme est un centre du monde. Félix Marcel Castan
Ainsi je suis responsable pour moi-même et pour tous, et je crée une certaine image de l'homme que je choisis; en me choisissant, je choisis l'homme. Jean-Paul Sartre

E ntre la fuite et la quête. Le mot «diversité » se rapporte à des domaines aussi différents que l'origine ethnique, la religion,

la culture, l'orientation sexuelle, les capacités physiques et mentales, les espèces animales et végétales. À son opposé, on trouve des termes comme « unitarisme», «nivellement», "protectionnisme», «standardisation du mode de vie », «banalisation des espèces». Cette opposition est ambiguë: l'humanité n'a jamais semblé aussi variée et la nature autant diversifiée. Jamais nous n'avons eu autant conscience de la fragilité de cette diversité. Jamais la typicité de chaque société et l'unicité de chaque être humain n'ont été autant affirmées. L'immunologie nous certifie le caractère absolument singulier de notre organisme, la biologie nous explique que notre code génétique est le nôtre et celui de personne d'autre, même les religions insistent sur notre relation particulière ou intime avec les forces surnaturelles, et davantage encore, notre cerveau demeure sans équivalent car l'individuation de chaque personnalité est un processus qui ne s'arrête qu'avec la mort. Aucun de nous ne peut donc penser comme un autre. L'uniformisation grandissante du monde tient, quant à elle, à autre chose. Ne sont-ce pas les aspirations humaines qui se ressemblent? Tous semblables parce que tous égaux ou du moins culturellement équivalents ou tous nivelés, invisibles, parce que la communication - ce bruissement intense qui tisse la trame du monde - brouille les pistes et apporte l'illusion du dialogue, quand les pays riches se barricadent dans leurs frontières et que s'établissent des traités de paix commerciale dictés par les économies les plus fortes? Les désenchantements sont à la mesure de cette solitude où se lisent la perte de l'altérité et la quête d'une «diversité culturelle».

\section{Patrimoine archéologique, patrimoine} orphelin. La préservation des sociétés humaines, traditionnelles notamment, et de leur histoire est indissociable d'une diversité qu'elles maintiennent, réduisent ou accroissent. Ainsi, la déclaration, accompagnée d'un plan d'application, adoptée par l'Unesco en 2002 à Johannesbourg, souligne que «la diversité culturelle et la diversité biologique ont la même signification et la même importance pour le développement durable ». D'autres pétitions internationales proposent une définition d'un patrimoine culturel immatériel ou patrimoine vivant comme « creuset de la diversité culturelle et garant du développement durable» (Unesco 2003). Par ailleurs, plusieurs conventions émanent du Conseil de l'Europe et permettent d'associer patrimoine culturel et patrimoine archéologique (en particulier, celles de 1955 à Paris et à Grenade, et celle de 2005 à Faro). ${ }^{2}$ Les sites archéologiques sont considérés 
« comme expression du patrimoine architectural », qui constitue lui-même « une expression irremplaçable de la richesse et de la diversité du patrimoine culturel » (Conseil de l'Europe 1955, préambule).

Ces textes n'ont pas les mêmes implications dans les États signataires, puisqu'ils émanent d'organismes supranationaux d'échelles différentes. Néanmoins, les définitions soulignent une réticence à lier les notions de diversité et de patrimoine culturels à celle de patrimoine archéologique, ce dernier n'apparaissant souvent que comme «essentiel pour la connaissance du passé des civilisations » (Convention européenne pour la protection du patrimoine archéologique de la Valette, dite Convention de Malte ${ }^{\mathbf{3}}$ ). Cependant, l'expression «patrimoine archéologique» et le mot «archéologie» ne figurent pas dans les conventions de l'Unesco précitées. On les trouve seulement dans des définitions générales hors convention, jamais au titre des patrimoines matériel et immatériel.

1 http://www.unep.org civil_society/PDF_docs/ Diversite_Culturelle_et_ Biodiversite.pdf);

voir également

la fiche d'information

No. 10 : « Populations

autochtones

et environnement",

téléchargeable sur http://

www.ohchr.org/french/

about/publications/docs/

indileaflet10_fr.doc,

et le programme 1999

des Nations Unies

pour l'environnement

(PNue ou UneP), Cultural

and Spiritual Values

of Biodiversity:

A Complementary

Contribution to the Global

Biodiversity Assessment,

http://www.unep.org

2 http://www.aidh.org/

Biblio/Txt_Europ/

autreso1.htm;

http://209.85.129.104/

3 http://conventions.coe.

int/Treaty/fr/Treaties/

$\mathrm{Html} / 143 . \mathrm{htm}$

4. On sait que le pillage

du musée de Bagdad

s'est produit à la suite

de la prise de la ville

par l'armée américaine;

on connaît moins

celui du musée

d'Hargeisha à la suite

de la destruction

de la capitale du

Somaliland par l'aviation

de Syad Barre.

5 Le pillage des pièces

archéologiques

et la destruction

de sites africains sont

une atteinte irréparable

à l'histoire de l'Afrique.

Une liste rouge

publiée par l'ICOM

(International Concil

of museums) est

téléchargeable sur icom.

museum/redlist/afrique/

french/intro.html).

En 2005, près de

800 pièces artisanales

et archéologiques

originaires du Mali

ont été saisies par

les douanes françaises à Montpellier.

des œuvres d'art antiques

sont vendues à

se rendant en Tunisie

et un trafic se fait jour

depuis quelques années

Roussillon via le Mali,

le Maroc et l'Italie.

Les agences de voyages

qui organisent des raids

porter une lourde

responsabilité

dans les vols de pièces

archéologiques

(El Watan, 25 novembre

2004 et 25 avril 2007). en Languedoc-
6 On trouvera un extrait

Hugo dans Demoule

7 On lira avec intérêt

Panalyse que fait de son

Hawass (Monsieur « $\mathrm{Z}$ »,

Sciences et avenir, janvier

2007, p. 36-39). de paysage, sur l'enrichissement paradoxal des haies au cours du temps et sur la nécessité de gérer ces espaces selon des procédures contraignantes relevant de l'agriculture traditionnelle (Marguerie et al. 2003). L'évolution du climat et les dynamiques de peuplement régional permettent d'aborder le rôle des sociétés dans la régulation ou le déséquilibre environnemental. Un cas exemplaire est létude des sites néolithiques du bord du lac de Chalain et de Clairvaux dans le Jura. La figure 1 illustre les «relations complexes qui lient les sociétés agricoles en permanente évolution et un environnement instable (Pétrequin et al. 2002). Cette «instabilité résulte de l'effet de processus naturels et (ou) du contrecoup des activités humaines. À l'échelle des phénomènes actuels, cette instabilité est inhérente au principe même du fonctionnement des systèmes, des réseaux et de l'évolution générale; elle ne doit pas nous étonner, si tant est que l'on accepte de faire abstraction de l'a-priori classique qui voudrait «opposer des sociétés traditionnelles, froides, à évolution lente, et des sociétés modernes, chaudes, caractérisées par des accélérations évolutives» (Van der Leeuw et al. 2003). Une grande partie des vestiges archéologiques sont encore enfouis et leur destruction sans étude constitue une perte pour la connaissance de la diversité des pratiques et du type de relations qui s'établissent entre les sociétés humaines et leurs milieux de vie (Lévêque et al. 2003). Les menaces qui pèsent sur cette diversité ne sont pas uniquement imputables aux destructions urbaines et rurales, mais sont également le fait des guerres ${ }^{4}$ et des dispersions dans des collections étrangères. ${ }^{5}$ Aussi, certaines nations, comme les pays de l'Union européenne, le Québec ou encore l'Algérie, se sont dotées d'une protection légale, en votant des lois garantissant la sauvegarde de leur patrimoine archéologique, considéré comme bien commun indépendant des intérêts privés. En France, on doit se féliciter de la loi de 2001 destinée à la prévention archéologique: «Une loi pour l'œuvre collective de nos pères », aurait dit Victor Hugo ». ${ }^{6}$ À l'inverse, un legs culturel n'est pas toujours socialement facile à intégrer.7 S'il est aisé pour les Français de s'identifier à un Gaulois moustachu, chevelu et râleur, il l'est moins pour un Antillais de se sentir Amérindien, pas plus qu'il n'est habituel, pour un descendant de colons de la ColombieBritannique, d'accepter l'héritage des peuples autochtones, généralement ignoré, détourné ou édulcoré.

Pourtant, la diversité des sociétés disparues fascine le public. Et si ses centres d'intérêt vont souvent vers les œuvres d'art et les architectures monumentales, les archéologues ne dédaignent pas non plus les trouvailles spectaculaires et les beaux objets dont ils illustrent leurs publications. Cependant, la majorité des découvertes archéologiques sont essentiellement constituées d'indices d'apparence modeste acquis au prix de recherches laborieuses (Demoule 2004). 


\begin{tabular}{|c|c|c|c|c|c|c|}
\hline $\begin{array}{l}\text { Dates } \\
\text { calendaires } \\
\text { av. J.-C. }\end{array}$ & $\begin{array}{l}\text { Nombre de } \\
\text { villages }\end{array}$ & Immigration & Acculturation & $\begin{array}{l}\text { Population } \\
\text { regroupée } \\
\text { à Clairvaux }\end{array}$ & $\begin{array}{l}\text { Population } \\
\text { regroupée } \\
\text { à Chalain }\end{array}$ & $\begin{array}{l}\text { Forêt } \\
\text { secondaire } \\
\text { jeune }\end{array}$ \\
\hline 2600 & & & CHALAIN & & & \\
\hline \multicolumn{7}{|l|}{2700} \\
\hline 2880 & & & $\begin{array}{l}\text { CLAIRVAUXX } \\
\text { RÉCENT }\end{array}$ & & & \\
\hline \multicolumn{7}{|l|}{2920} \\
\hline 2970 & & & $\begin{array}{l}\text { CLAIRVAUX } \\
\text { MOYEN }\end{array}$ & & & \\
\hline 2990 & & FERRIERES 3 & & & & \\
\hline \multicolumn{7}{|l|}{3000} \\
\hline \multicolumn{7}{|l|}{3020} \\
\hline 3030 & & & $\begin{array}{l}\text { CLAIRVAUX } \\
\text { ANCIEN }\end{array}$ & & & \\
\hline 3040 & & $\begin{array}{l}\text { FERRIÈRES } 2 \\
\text { CLAIRVAUXX }\end{array}$ & & & & \\
\hline \multicolumn{7}{|l|}{3060} \\
\hline 3100 & & & & & & FRÊNE \\
\hline 3200 & & $\begin{array}{l}\text { HORGEN et } \\
\text { FERRIËRES } 1\end{array}$ & HORGEN & & & \\
\hline 3300 & $\begin{array}{l}\text { Longue } \\
\text { interruption } \\
\text { de l'habitat }\end{array}$ & & & $\begin{array}{l}\text { Longue } \\
\text { interruption } \\
\text { de l'habitat }\end{array}$ & $\begin{array}{l}\text { Longue } \\
\text { interruption } \\
\text { de l'habitat }\end{array}$ & \\
\hline
\end{tabular}

[Fig.1] Corrélation entre densité

de population, immigration, état

du milieu forestier, alimentation

carnée et climat des sites de Chalain

et Clairvaux (Jura), entre 3300 et

2600 av. notre ère (d'après Pétrequin

et al. 2002). 


\begin{tabular}{|l|l|l|l|l|l|}
\hline $\begin{array}{l}\text { Forêt } \\
\text { primaire }\end{array}$ & $\begin{array}{l}\text { Forêt secondaire } \\
\text { jeune et âgée }\end{array}$ & $\begin{array}{l}\text { Litères } \\
\text { de frêne }\end{array}$ & $\begin{array}{l}\text { Litères } \\
\text { d'orme }\end{array}$ & $\begin{array}{l}\text { Litères } \\
\text { de sapin }\end{array}$ & $\begin{array}{l}\text { Maximum } \\
\text { de la chasse } \\
\text { au cerf }\end{array}$ \\
\hline
\end{tabular}

\begin{tabular}{|l|c|c|c|c||}
\hline & HÊTRE & & \\
\hline & & & \\
\hline
\end{tabular}

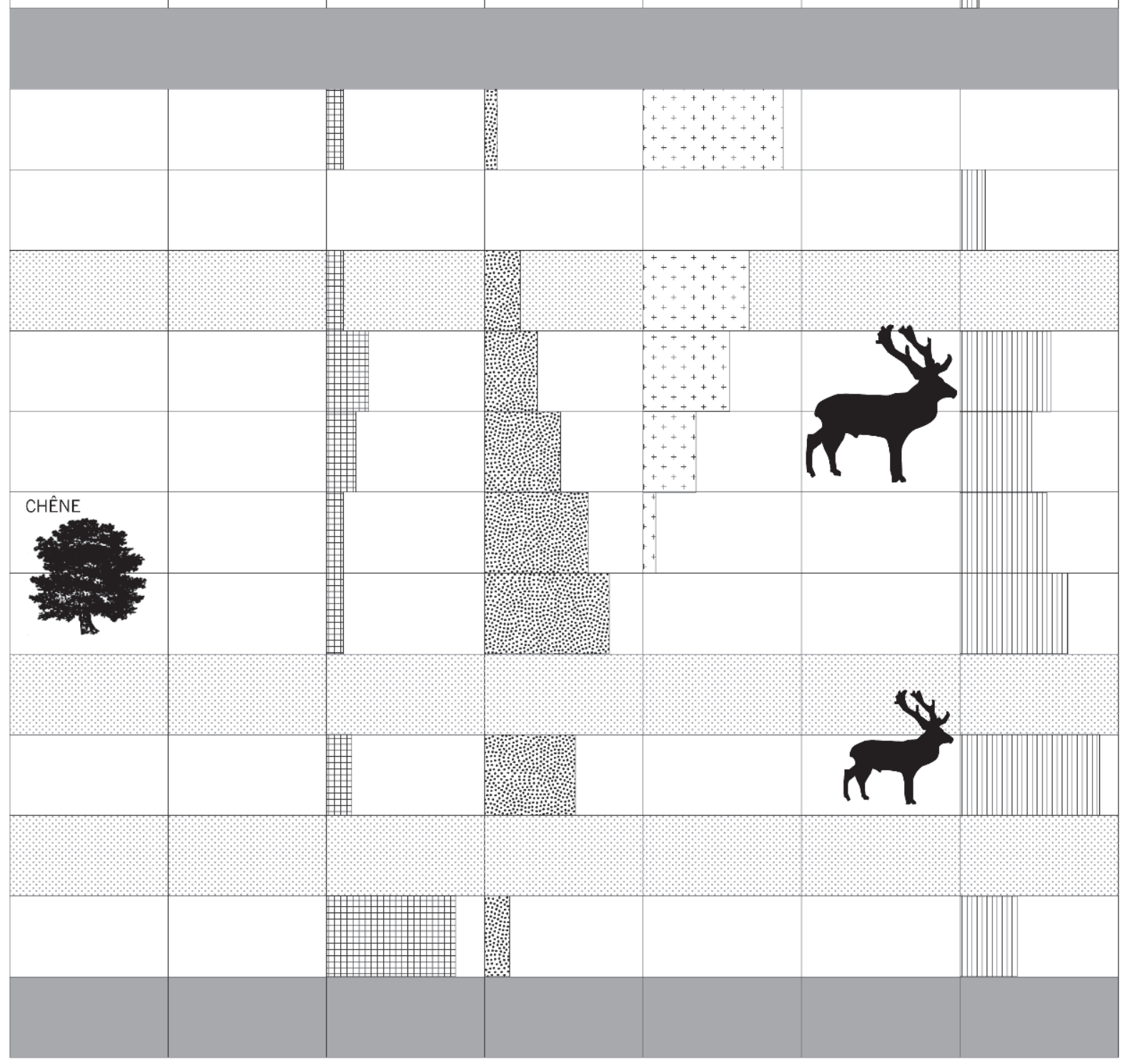

Dégradation climatique majeure

Dégradation climatique

majeure 


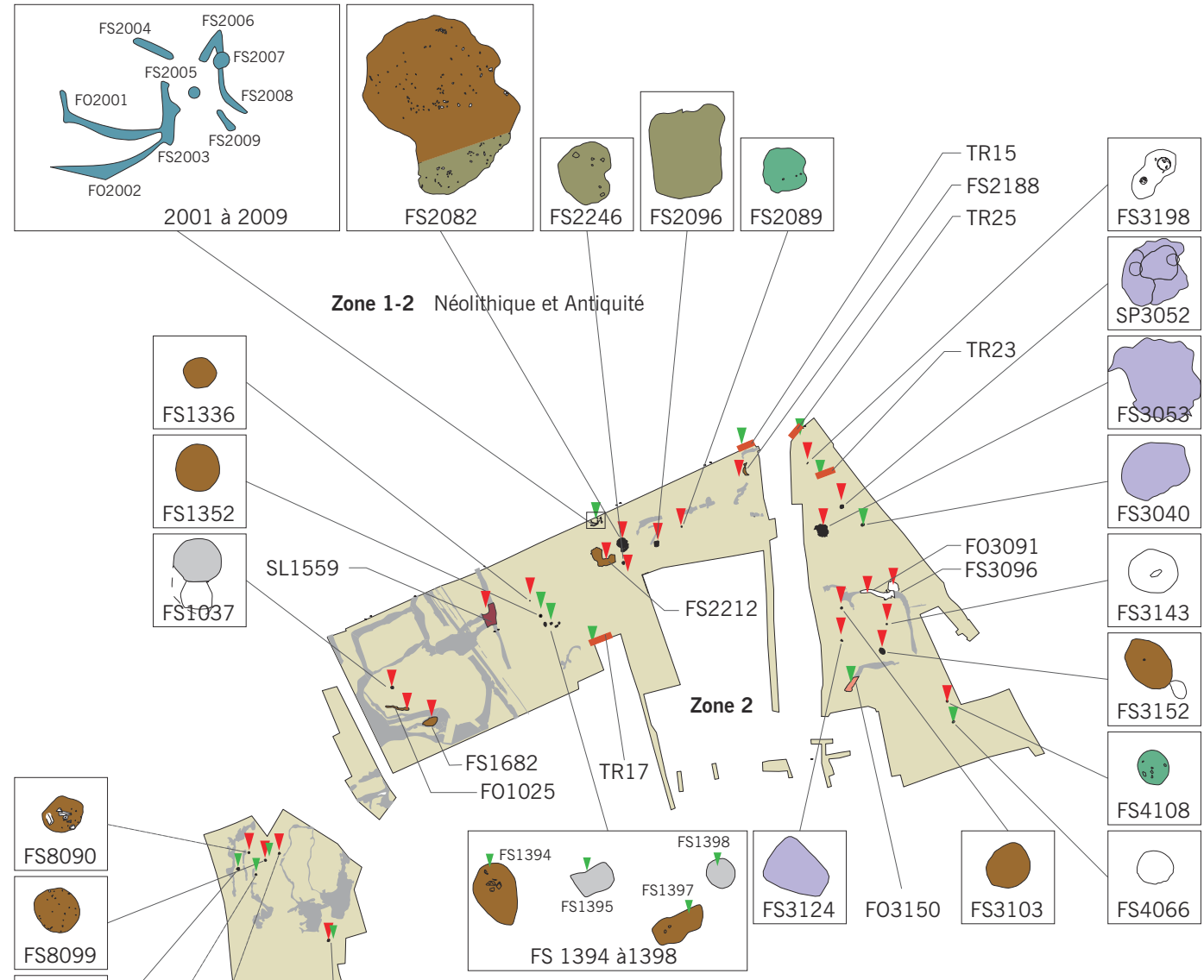

Zone 3-4 Néolithique, Bronze final et Antiquité

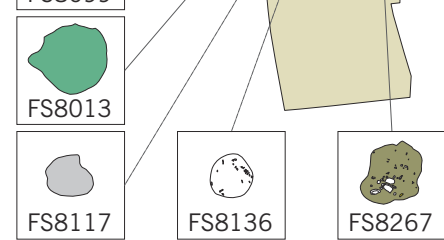

Zone 8 Néolithique et Bronze moyen

Zones de fouille

- Relevé géomorphologique

$\nabla$ Prélèvement sédimentologique

$\checkmark$ Prélèvement micromorphologique
Chasséen probable

Chasséen

Epi-Ferrières

Néolithique indéterminé

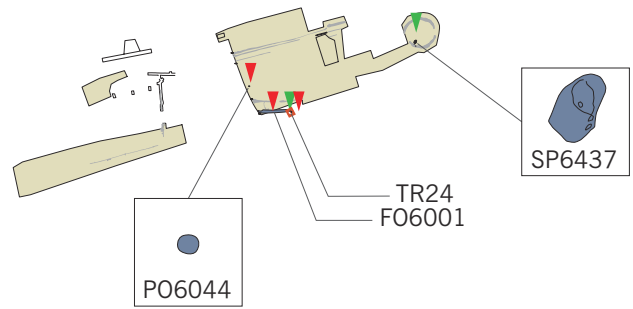

Zone 6 Âge du Fer et Antiquité

- Campaniforme

Campaniforme et Fontbouisse

Bronze final

Âge du fer

- Antiquité gallo-romaine
[Fig.2] Implantations des prélèvements sédimentaires et micromorphologiques sur le site

de Mas de Vignoles IV (Nîmes, Gard). Cette stratégie illustre la contribution interdisciplinaire dans le cadre d'une fouille préventive et les diverses échelles de résolution nécessaires à toute interprétation.
[Fig. 3] Peintures de l'abri 1 de Las Geel (Dhubato, République du Somaliland). Il s'agit d'un nouveau style d'art rupestre jusqu'alors inconnu en Afrique de l'Est.

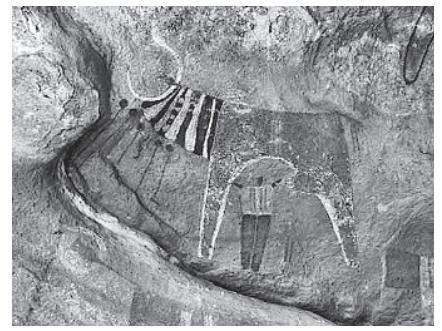


Nous rappellerons ici l'autopsie d'Ötzi, l'homme congelé dans les glaces du Tyrol. Ce n'est pas l'aspect extraordinaire de la découverte qui est intéressant, mais les indices ténus relatifs à la composition des objets, à la fabrication des vêtements et au contenu des viscères, l'analyse des pollens et des résidus divers, les mesures radiométriques et spectrométriques.

En archéologie, il n'y a pas de grandes et de petites découvertes. La diversité des données, à différentes échelles d'observation, est une nécessité de la recherche; elle implique à la fois la collaboration des disciplines et la confrontation des résultats [Fig.2]. Entre la spore et la tombe, ce qui fait sens réside dans l'enchaînement des événements qui ont conduit à découvrir l'une et l'autre. Chaque élément apporte sa part de témoignage et sa temporalité.

Il est évident que la diversité culturelle se manifeste de manière bien plus chatoyante à travers la diversité des cultures vivantes. «En Afrique, à chaque fois qu'un vieillard meurt, c'est une bibliothèque qui brûle», déclarait Amadou Hampâté Bâ il y a près d'un demi-siècle à la tribune de l'Unesco. Ce grand homme se disait «religieux, poète peul, traditionaliste, initié aux sciences secrètes peules et bambara, historien, linguiste, ethnologue, sociologue, théologien, mystique musulman, arithmologue et arithmosophe». Nous avons assurément beaucoup à apprendre des peuples et des cultures que nous croisons dans nos cheminements archéologiques, tout en sachant que l'archéologie peut conduire les populations autochtones à découvrir une histoire dont le colonialisme les a souvent privées. J'évoquerai ici l'exemple de la jeune république du Somaliland (ancienne colonie britannique située au nord de la Somalie), qui est depuis 1991 en quête d'une identification internationale. Une équipe française, qui comprend des chercheurs de l'université de Montpellier, du ministère de la Culture, du CNRS et de l'Inrap, y a révélé à mi-distance entre la capitale Hargeisa et le port de Berbera - des peintures rupestres à l'esthétique remarquable. Datées du III millénaire avant notre ère (Gutherz et al. 2003), elles étaient pratiquement inconnues des habitants des lieux [Fig.3]. Cet art d'une richesse exceptionnelle a permis au pays de s'afficher comme le détenteur d'une histoire riche et ancienne. Il a contribué à faire apparaître le Somaliland sur les cartes de l'Afrique. À la demande du gouvernement de ce pays, l'équipe a commencé à répertorier les vestiges d'époque plus récente, afin d'aider

à la restitution d'une préhistoire et d'une histoire encore largement méconnues. Ces découvertes ont aussi engendré quelques actes de vandalisme et le début d'une activité touristique. Il a donc fallu participer à l'élaboration d'une législation, collaborer à la protection et à la mise en valeur des sites rupestres, et proposer une formation en archéologie à destination des étudiants nationaux. Face à l'art de Las Geel, les Somalilandais, d'abord étonnés, ont éprouvé une réelle fierté, sans refuser toutefois de s'interroger sur la nature des connexions entre leur histoire récente, le déterminisme religieux et leurs origines culturelles. Cet exemple illustre bien la part d'emprunts et d'échanges qui nourrit toute culture. L'archéologie participe évidemment à cette négociation permanente, car la méconnaissance ou la destruction des vestiges du passé réduisent la diversité et privent les cultures d'une dimension identitaire essentielle.

Diversité pour un futur. Le rapprochement entre diversité culturelle et archéologie n'implique pas seulement des problématiques scientifiques et institutionnelles, mais traite aussi d'éthique et du principe démocratique. Dans ce dispositif, la place de l'archéologie n'est pas anodine. Ses découvertes possèdent en effet une puissance légitimatrice qui peut étonner les archéologues; ces derniers contribuent pourtant à reformuler la notion de diversité culturelle. Par ailleurs, les rapports «société-milieu » sont aujourd'hui au cœur des questionnements archéologiques et des interrogations écologiques que se posent dramatiquement les sociétés du XXI ${ }^{\mathrm{e}}$ siècle. Ainsi, l'action des archéologues dépasse les limites des revendications particulières pour restituer la diversité des cultures humaines, rétablir le rythme lent des parcours de l'humanité et participer à l'élaboration d'un projet planétaire stimulant.

CAneva I. 2001: « De Cayönü à Mersin: les premiers villageois de l'Anatolie du sud-est», in J. Guilaine (dir.), Communautés villageoises du Proche-Orient à l'Atlantique (80oo-20oo avant notre ère), Séminaire du Collège de France, Paris, Errance, p. 13-32.

Cauvin J. 1994: Naissance des divinités, Naissance de l'Agriculture. La révolution des symboles au Néolithique, Paris, CNRs Éditions.

Conseil de l'Europe 1955: Grenade (Espagne), Convention pour la sauvegarde du patrimoine architectural de l'Europe; http://209.85.129.104/search?q=cache:LHUiBoqmAFgJ:www. nikekultur.ch/fileadmin/user_upload/PDF/Charten/granada_f. $\mathrm{pdf}+$ sauvegarde+du+patrimoine

Demoule J.-P. (dir.) 2004: La France archéologique: Vingt ans d'aménagements et de découvertes, Paris, Hazan.

Demoule J.-P. 2005: L'Archéologie entre science et passion, Paris, Gallimard, «Découvertes».

Demoule J.-P. 2007a: Naissance de la figure, Paris, Hazan.

Demoule J.-P. (dir.) 2007b: L’Archéologie préventive dans le monde: apports de l'archéologie préventive à la connaissance du passé, Paris, La Découverte.

Gutherz X., Cros J.-P., Lesur J. 2003 : «Nouvelle découverte de peintures rupestres dans la Corne de l'Afrique: les abris sous-roche de Las Geel, République de Somaliland », Annales d'Éthiopie, vol. XIX, p. 295-306.

JALLOT L. 2004: « Mas de Vignoles IV à Nîmes (Gard) », in L. Jallot (dir.) Le Mas de Vignoles IV à Nîmes (Gard), DFs, Afan, SRA LanguedocRoussillon, 2 vol., 13 fascicules, vol.I: «Géoarchéologie, les données environnementales et sédimentaires - Méthode et organisation des équipes ", p. 5-10.

Lévêque C., Muxart T., Abbadie L., Weil A., Van der Leeuw S. 2003: «L'anthroposystème : entité structurelle et fonctionnelle des interactions sociétés - milieux », in Ch. Lévêque et S. Van der Leeuw (dir.), Quelles natures voulons-nous?, Paris, Elsevier, p.110-129.

Marguerie D., Antoine A., Thenail C. et al. 2003: «Bocages armoricains et sociétés, genèse, évolution et interactions », in T. Mauxart, F. D. Vivien, B. Villalba, J. Burnouf (dir.), Des milieux et des hommes: fragments d'histoires croisées, Paris, Elsevier, «Environnement», p. 115-131.

Pétrequin P., Arbogast R.-M., Magny M. 2002 : «Premiers agriculteurs néolithiques à effet-retard: Chalain et Clairvaux (Jura) du XXXIII ${ }^{e}$ au XXVIII ${ }^{e}$ siècle avant J.-C.», in T. Mauxart, F.D. Vivien, B. Villalba, J. Burnouf (dir.), Des milieux et des hommes: fragments d'histoires croisées, Paris, Elsevier, «Environnement», p. 57-64. 
Richard H., VignOt A. (dir.) 2002 : Équilibres et ruptures dans les écosystèmes depuis 20 ooo ans en Europe de l'ouest, Colloque international, Besançon, septembre 200o, Paris, Les Belles Lettres, p.191-203. (Annales littéraires de Franche-Comté, série Environnement, Société et Archéologie 3).

UNESCO 2003 : Paris: Convention pour la sauvegarde du patrimoine culturel immatériel, 17 octobre 2003 ; http://www.unesco.org/ culture/ich/index.php?pg=00022

VAN Der Leeuw S., Favory F., Fiches J.-L. (dir.) 2003: Archéologie et systèmes socio-environnementaux: Études multiscalaires sur la vallée du Rhône dans le programme Archeomedes, Paris, CNRS (monographie du Cra).

\section{En marge des femmes : la société contre le désir des hommes}

\author{
Pierre Lemonnier \\ CNRS-Credo
}

La question n'est donc plus: comment le pouvoir émerge-t-il? Mais plutôt: pourquoi les mécanismes de contrôle d'une émergence excessive du pouvoir font-ils parfois défaut? Jean-Paul Demoule (2007, p. 88)

$\mathbf{P}$ lus discret sur les conditions d'apparition de l'État (1974, p. 161-186) que dans son ahurissante détestation des marxistes (1980 [1978]), Pierre Clastres défendit, dès 1969, l'idée que les sociétés « ont très tôt pressenti que la transcendance du pouvoir recèle pour le groupe un risque mortel» (1974 [1969], p. 40). Selon l'auteur de La Société contre l'État, ce fut la mise en place du couple coercition-subordination, cette « essence du pouvoir partout et toujours » (ibid., p. 12, souligné par Clastres), qui conduisit aux «sociétés historiques [...] qui portent en elles la cause de l'innovation, du changement, de l'historicité » (ibid., p. 22, souligné par Clastres). Appuyée sur le solide portrait d'un leader politique amazonien dépourvu d'autorité, cette théorie d'une limitation du pouvoir par les sociétés locales n'a pas manqué de faire école. Chez Marshal Sahlins (1963), sans doute, dont le big man mélanésien ressemble comme un frère au chef «contraint, sous peine de se voir rapidement abandonné par la plupart de ses gens, de tenter de satisfaire leur demande»(Clastres 1974, p. 35), et jusqu'à Jean-Paul Demoule, qui consacre trois paragraphes de son dernier ouvrage aux divers moyens, pour une société, de «se prémunir contre la montée de pouvoirs trop forts» (2007, p. 89).

La société, bien entendu, n'est contre rien du tout car elle n'existe qu'à travers des pensées individuelles, fussent-elles inscrites en institutions ou mises en gestes et en objets. En revanche, la recherche de l'équité conduit parfois les humains à créer des enchaînements de pratiques dont les effets sociaux et matériels, aussi inattendus qu'incontrôlables, sont à l'opposé de l'objectif recherché. C'est cette dimension inintentionnelle et paradoxale des logiques sociales que j'aborderai ici, à partir de l'exemple de sociétés de Mélanésie.

Fanatiquement égaux. À côté de "chefs » contrôlant la vie de tous à coups d'interdits et ayant jadis droit de vie et de mort sur leurs sujets, la Mélanésie comporte (-ait) nombre de personnages politiques auxquels les observations de Clastres sur l'équité dans les sociétés préindustrielles s'appliquent spectaculairement. Des Anga de Papouasie Nouvelle-Guinée, on dirait certainement que «l'idée de donner un ordre ou d'avoir à obéir» leur est «quelque chose de tout à fait étranger» (1974, p.11-12). Et l'autorité temporaire de leurs grands guerriers s'accorde tout autant à cette constatation que «le modèle du pouvoir coercitif n'est [...] accepté qu'en des occasions exceptionnelles, lorsque le groupe est confronté à une menace extérieure » (ibid., p. 27).

Surtout, beaucoup de sociétés mélanésiennes ont elles aussi ce «goût de l'égalité» repéré par Clastres (ibid., p. 26) et déploient des efforts spectaculaires pour défendre la parité des personnes et des échanges. Cela était (ou reste) particulièrement explicite sur la côte sud de la grande île, où s'appliquait (s'applique) un strict principe d'équivalence (en nature et en quantité) dans les domaines les plus variés: se marier par échange de sœurs (réelles ou classificatoires), tuer pour venger un assassinat ou une perte à la guerre, infliger à un agresseur la même blessure que celle qu'il a causée, payer une contremagie le prix qu'a coûté l'acte de sorcellerie qu'elle est censée annuler, échanger une femme contre une femme, divorcer parce que la sœur que l'on a donnée en échange de sa propre femme se sépare de son mari, prêter son épouse contre celle de son amant d'une nuit, rendre autant que l'on a reçu, etc. (Lemonnier 1993).

\section{La société contre les idées reçues : exploitation} et despotisme chez les big men. Nombre de groupes de Mélanésie n'en ont (ou n'en avaient) pas moins des leaders dont le statut social est bien plus qu' «enviable» (Clastres 1974, p. 35) et comporte la faculté de se faire obéir et d'exploiter les autres, et d'abord leur travail, y compris les big men, qui constituent à juste titre la version mélanésienne de ces «fœetus culturels du pouvoir [...] voués à périr» (ibid., p.17). Bien que présentés comme des personnages politiques situés à l'écart du chemin- qui-mèneà-la-chefferie-voire-à-l'État grâce à des mécanismes sociologiques autorégulateurs s'opposant à l'accumulation de pouvoir, les big men ne se privaient pas d'exploiter autrui, comme l'indique le triste sort de ces rubbish men décrits par Meggit (1977, p.190-191) chez les Mae-Enga. Lors d'une redistribution de biens par un big man, ces «pas grand-chose»-qui ont aidé le haut personnage à rassembler les biens (porcs, coquillages) offerts par lui au nom de tous lors d'un précédent échange compétitif viennent demander leur part. Mais, loin de voir leur revendication satisfaite, ils sont éconduits puis frappés par d'autres protégés du big man. Non seulement le big man mélanésien prospère en convainquant les autres de lui fournir gratuitement leur travail (pour élever les porcs 